\title{
RFID and Evidence Based Stock Management
}

\section{Catherine Cooke}

I should like to thank the Technische Hochschule for giving me the opportunity to talk about what we've been doing with Stock Management and RFID hand-held scanners. Much of this presentation is inevitably about the systems we use in Westminster - SirsiDynix Symphony and Intellident RFID scanners - other products are different.

I should perhaps explain a little about Westminster Libraries. We are the public library service for the City of Westminster, one of the 33 London boroughs and one right at the heart of London. We have 11 libraries, 2 satellite Libraries, an Archives and Local History Centre and a Home Library Service. We have nearly 3 million visits a year and a membership of around 87,000 with a $30 \%$ annual turnover. As a central London authority we have a relatively small resident population, but a very large daytime population. We hold some 1 million items in stock, with about 2.5 million items loaned annually. There are 195 staff (140 full time equivalent posts). We have some of the longest opening hours in London. Our earliest opening is 7.30am at Pimlico Library, a new library which shares its building with a school and Adult Education Centre, offering services to them as well as serving as the local library for Pimlico. Our latest closing is $10 \mathrm{pm}$ at Paddington Library. 5 of our libraries are open on Sundays.

While we do have long-standing members, much of the population is transient - they come and live in Westminster for a year or two, then move on. Our membership and visitor figures have been generally rising over the last 5 years and this at a time when national trend has been that they are dropping. We run customer satisfaction surveys every couple of years and the results show the service as good and improving - $90 \%$ of our users say the service is Good or Very Good.

Westminster Libraries are very busy and active with relatively few staff, especially in the traditional »back office« areas such as Acquisitions.

We have been using RFID for some 4 years now, implementing Intellident equipment from 2007 first in our 4 largest libraries. As a general rule we have 3 self service machines in each library offering issue, renewal, discharge and payment, as yet taking coins only. We regarded it as a transformation of the service because as self-service went in we, as others have already mentioned they did, removed the big old counters and installed smaller staff desks away from entrance. When you enter the library the first thing you see is the self-service - the counter is further back. Staff are no longer behind counters but out in the library floor-walking in a similar way to shop staff. They take customers to self-service and train them. Our non-book material, DVDs and CDs, are open access. 
Westminster is a relatively small geographical area - you can travel by public transport from a library at one end to one at the other in about half an hour. Many customers use more than one library. We therefore tagged all lending stock at the beginning of the implementation, the work being done by temporary staff working in teams round the libraries. All staff stations were equipped with RFID pads and software. Over the next couple of years, as libraries were refurbished or, in the case of Pimlico, relocated, we took the opportunity to introduce self-service. All libraries are now fully self-service.

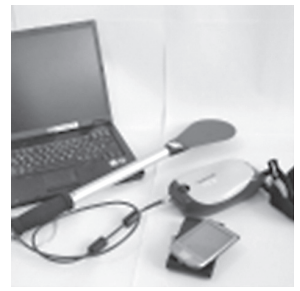

With the introduction of RFID, stock items no longer have a date label to indicate their last issue date or how often they are used. Both the self-service kiosks and any staff issues give the customer a receipt with the due date on it - plus it acts as a bookmark!

I am including a few examples of scanners available in England this is one from Bibliotheca.

So how does one approach Stock Management in this day and age? The traditional way when I started years ago was to look at the date labels. We stopped using date labels when we moved to RFID for self-service and staff issue - we give a receipt instead

You could run reports off the LMS. Standard reports are available on Symphony which could extract the data, or you could use an external Reporting Tool such as Crystal Reports or Cognos Impromptu. Both these methods, however, require staff with a significant understanding of the database structure to set up and run reports and they are time consuming.

We use Evidence Based Stock Management software, a system called SmartSM from the company BridgeAll. There is an automated monthly data extract from Symphony to a separate database system. This is scheduled at $9.15 \mathrm{am}$ on the 1 st of every month and takes just under half an hour. The extracted file is automatically sent by ftp to BridgeAll, where it is automatically loaded into SmartSM and made available. The result is presented in a web interface with pre-designed tailored reports. We made decisions centrally about the parameters used in these reports and they can be changed for specific reasons, or for specific sites where different parameters would better suit the library. The system is easy for staff to use with minimal training and there is no impact on the live Symphony system.

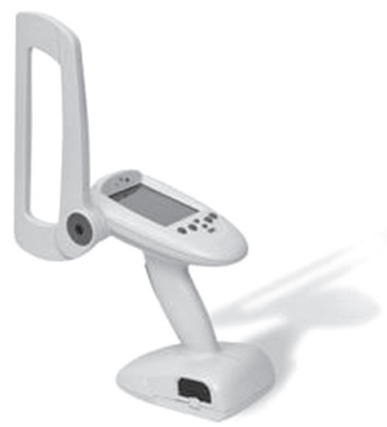

Lists of stock can be printed off and staff search manually for the items - that is how we started. Now there is also an option to download the reports to a hand-held scanner - such as to this example from 3M. Again there is a parameter to specify which company's scanner is in use in the authority.

We've concentrated on two areas of SmartSM using Stock Management software - there are other facilities within it. 


\section{Dead Lists}

Dead Lists - items that have not been issued for a significant period of time. It is up to the library to decide what they regard as significant. A fiction book not issued in over 6 months might be considered 'Dead' and should be removed. For non-fiction, you might give it 1 year. We are a public library. One of our main success indicators is the number of issues. If an item is not earning its place on the shelves, it should be removed and replaced with a newer, better, more attractive item that will earn its place. I should add that we do keep material for more than this, classics such as Dickens' work for instance. Check the shelves, delete those items you find you don't want to keep, ISSUE and DISCHARGE those you want to keep so they don't come up as unused again for a while and finally delete everything you can't find.

\section{Grubby Lists}

Grubby Lists - items that have been issued a certain number of times. A book that has been issued 25 times might be considered 'Grubby' and should be removed. If it's a children's book, you might think 15 or 20 times would be enough to wear it out. These items are popular and still being used -

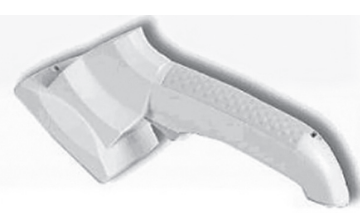
maybe one grubby hardback should be replaced with 2 bright new paperback editions. This is an alert to items to consider, it is not a categorical instruction to delete something. You might decide to keep it because there are no paperback versions of that title on the shelves, it is still in good condition or it is out of print and impossible to replace. Check shelves for books, delete those you find you don't want to keep, consider reserve stock for those you feel worth keeping but that aren't really in good enough condition for the open shelves, and again, delete everything you can't find. This is the scanner from D-tech.

This is the Intellident scanner we are now using. RFID is often seen as self-service, but it is potentially much more.

The delivery of original hand-held scanners was in mid-Oct 2008. They were a different, bigger design and there were a few teething problems, particularly around the battery life of hardware. You could scan for about 2 hours, then you had to recharge them overnight before you could do anything else. New equipment was released which we purchased about 18 months ago - this one. It's a Nordic scanner with a multi-directional antenna designed in UK by Intellident. You can use it in this orientation if you are scanning books shelved normally, or twist it through 90 degrees and down to scan racks of DVDs or CDs. This means you can easily keep the antenna in the same orientation as the tags, but still hold the scanner comfortably and see the screen. The battery life is much

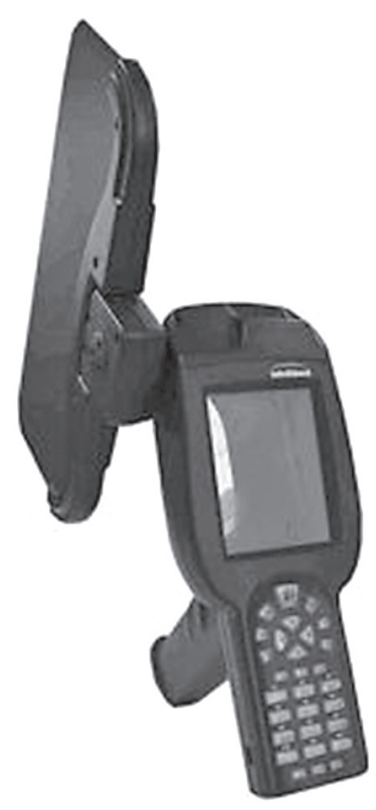


better - 6 to 8 hours - and you can upload and download reports while recharging. It sits in a cradle connected to the PC by USB when not in use. This is the one we are using now.

As these are stock functions, they are largely bound up with Symphony which has three processes for RFID stock work:

\section{Shelf tidy}

Shelf tidy - This is not an immediate priority for us. We are a public library and do not use long Dewey classification numbers. Stock is shelved in broad subjects like Science or Photography and staff can tidy shelves by eye quite easily.

\section{Search}

For this you run a program on Symphony or download a file from SmartSM, then download the result to the hand-held unit via a PC. You scan the shelves, recharging as necessary. The scanner bleeps to alert you when it finds an item and displays brief details on the screen so you can take it off and deal with it there and then or put it to one side to deal with later. You then upload the result to Symphony and run another program to update the catalogue. If you search for items with a particular status, perhaps with reservations or a status like In Processing, those not found are marked MISSING by the report processing the result file.

\section{Inventory}

For this you take a blank hand-held scanner and scan shelves to capture the items. You upload the resulting file to Symphony and run a program to process it. For every item found on the shelves, the program enters the date you run this program in an Inventoried field on the records of items. Items not scanned on the shelves are left with NEVER or an old date in the Inventoried date.

The first case study was done with the old scanner. I searched Marylebone Library Adult stock for over 1,000 MISSING items, a process which took 5 hours. We mark anything we cannot find as MISSING, usually as the result of looking for it for a reservation. Marking it MISSING allows the LMS to try the next library that should have a copy, and prevents time being wasted looking for the same thing in the future. Staff do not, however, have time to go and look again several weeks later for these items, so unless someone happens to borrow them, they tend to stay marked MISSING.

In a second pass I inventoried all Marylebone Adult Fiction in about $31 / 2$ hours, which gives an average of 1,500 items per hour - something absolutely impossible by traditional methods.

Books shelved normally and standing face on read well. Those lying flat on metal shelves do not - the tag is too close to metal. You just pick the book up and pass the scanner underneath it - it takes less than a second. 
The scanner has a broad field - reading in advance of the antenna and above and below as well, so as you scan one shelf and back along the next, the scanner has a couple of opportunities to read tags. I also did some tests with programmed tags stuck on cards scattered on a desk, even partly piled up. The new scanner showed $100 \%$ read rate with the scanner passed over them at a moderate speed. It is not so good if you go too fast.

Using a program on Symphony to create a search file takes time, maybe up to an hour to define and run it, being clear about matters such as which Library, Item Type, Item Category, Status/s to include/exclude and which date parameters you want. Data transfer between the scanner and the PC and between the PC and Symphony is very simple and fast, a matter of seconds only.

Processing the results of a search or inventory does take time though, about an hour for every 2,000 items. After an inventory, Symphony reports the barcode, shelf mark and brief author and title details, plus the date of issue for items reported on loan, so you investigate those before the date of the scan, which is useful when you are building up a scan over several days. You can then do a manual search for various items reported on loan, in transit, at a library other than the library scanned or those that did not match in the catalogue (the report gives the identity of an item scanned near the one that could not be matched).

Deleted items do seem to find their way back on to the shelves from time to time. I suspect a lot of what was on the shelves that shouldn't have been was down to customers not putting items where the self-service kiosks tell them to, especially in the early days of self-service. We do not have sortation units - we do not have the space in our libraries and our throughput is not high enough to justify them. Our kiosks do a basic sort. If an item requires attention - transit to another library, is reserved or a DVD or CD whose case needs relocking - the kiosk displays a big red arrow pointing to a closed box. If an item should just be shelved, it displays a big green arrow pointing in the other direction to a trolley. I have watched someone when the kiosk displayed a red arrow put the item on the trolley the other side, and when it displayed the green arrow, put the item in the closed box! We do not check the items on trolley. If someone else has not already borrowed something by the time we deal with the items on the trolley, we just reshelve them.

Case study 2 was done using a Grubby Stock list from SmartSM. I pulled off Marylebone Grubby »Hardback Fiction« to my PC, connected the handheld and transferred the file, a process which took about 5 minutes. This is to be compared with about an hour using Symphony to crate the file and had no effect on the System - it doesn't risk slowing it and you don't have to avoid other reports that run at specific times.

It took me 1 hour 40 minutes to search Marylebone's Adult Fiction for 248 items. I found 150. Of the remaining 72, 64 were on loan, 1 on the hold shelf and 7 marked Missing. This is a rate of $1 \frac{1 / 2}{2}$ items a minute - absolutely impossible searching by eye. Indeed it was so fast that I had to take the scanner away from the shelves from time to time as I couldn't pull the books off as fast as it was finding them. In fact, the Library Manager was 
quite interested and pulled a book off the shelves a few feet in front of me that she felt was grubby. We held it to the scanner and it bleeped - it was one of the books it wanted.

I uploaded the results file to Symphony, the first time I had started from SmartSM and uploaded back to Symphony. It reported the right numbers and listed all the items on loan or Missing. It inventoried found items as expected. As it was a very small file, it didn't take long to run.

Pulling a file like this from SmartSM is vastly quicker and easier than creating the report on Symphony. It opens the process to anyone we can give access to SmartSM without any impact on the system. We do not let just anyone have access to Symphony reporting - only a few trained staff. Searching by eye for items on a report like this is not easy and very frustrating as $44 \%$ of items included shouldn't be on the shelves in the first place being legitimately on loan. I do feel is worth checking for items marked Missing or on loan - I found quite a lot of both on the shelves.

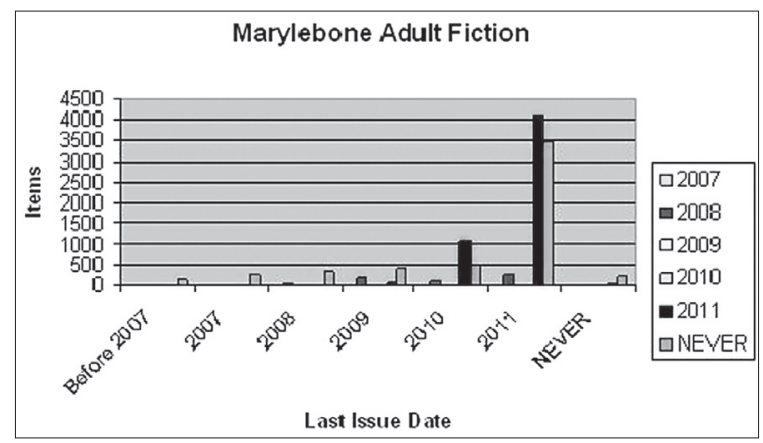

The coloured bars are the year when items were inventoried, set against a range of Last Issue Dates. Looking at the results in this graphical form, we can see there is a small amount of material that has not been issued for some years and NEVER inventoried. This may be accounted for by the fact that Symphony retains details if items with fines owing on them, even though the item itself has been physically withdrawn.

The third case study is a very recent one and I am still analysing the results. It was our first properly conducted inventory using a scanner. I should say that we have not run a proper catalogue clean-up for about 4 years.

There are three elements to an inventory:

- Run a program to mark all items currently out on loan as inventoried with the run date as the inventory date. This is the start date, in this case 1st June 2011.

- Run a program early every morning to mark as inventoried all items issued the day before.

- Scan the stock on the shelves.

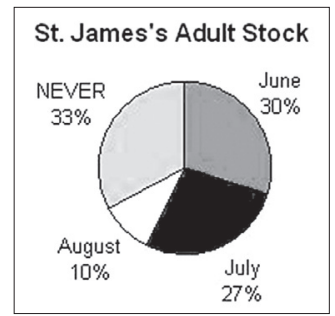

This means you only have the one field to worry about - the Inventoried field. At the end of the three months, anything that has either NEVER or a date before the start date in the Inventoried Date probably no longer exists and can be removed from the catalogue.

The pie chart shows the percentage of stock inventoried each month. I have to admit that $33 \%$ NEVER inventoried seems 
rather high. It may be that staff did not complete the Adult stock in the limited time available for this project and stock already marked MISSING may be included. On the other hand, another London authority did a similar exercise in their Central Library recently using another company's scanners - they found $25 \%$ of the stock did not get inventoried. Again this is probably higher than you'd expect. It raises questions about what happens to stock, questions probably better investigated in due course once an initial complete inventory has been done for the first time in many years. There's too much unknown over too long a period at present.

$\begin{array}{rrr}\text { Last } & \text { Inventoried } & \text { Inventoried } \\ \text { Issue } & 2011 & \text { NEVER } \\ 1999 & 0 & 48 \\ 2000 & 0 & 14 \\ 2001 & 0 & 50 \\ 2002 & 0 & 48 \\ 2003 & 1 & 51 \\ 2004 & 1 & 144 \\ 2005 & 27 & 106 \\ 2006 & 398 & 139 \\ 2007 & 477 & 759 \\ 2008 & 563 & 752 \\ 2009 & 304 & 2016 \\ 2010 & 1467 & 3076 \\ 2011 & 15130 & 3395 \\ \text { NE VER } & 39 & 920\end{array}$

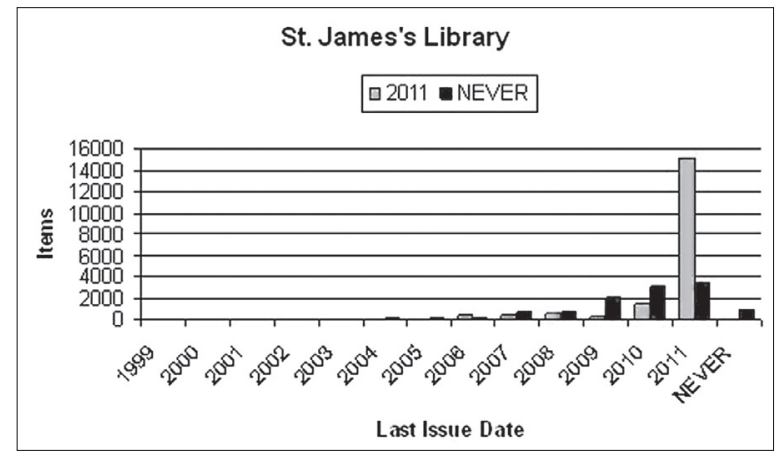

This is looking at this stock broken down by Date of Last Issue. If something has not been out for years and was not picked up in the Inventory, it probably does not exist. For instance, 27 items last issued in 2005 were inventoried on the shelves; 106 were not - the 106 probably no longer exist. In fact we'd probably also wonder if the 27 are worth keeping since no one seems to want to borrow them!

You can also drill down to specific stock areas to see the proportion of stock NEVER inventoried in particular areas. This shows our various Fiction categories - paperback, hardback, crime, romance etc. St. James's Library is a small library, so there are only a few items in some of the categories, so the percentages can be a bit misleading. This merely shows that

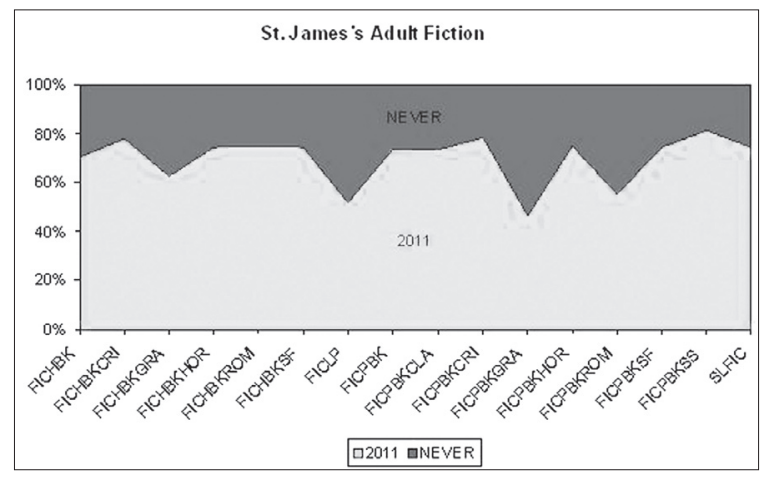
once you have the data collected and uploaded to the catalogue, you can look at it in different ways.

We have 7 hand-helds and 11 libraries, so we will probably base them at the larger libraries and they share them with the smaller ones. We also need to install the relevant Intellident software on PCs, creating the necessary file structures. We in Libraries are not allowed to do this and must wait for our Corporate IT support people to do it for us. We also need to add the Symphony utilities and reports to specific user profiles so they can upload data from the scanners to update the catalogue. Staff generally do not 
have the permission to run reports on Symphony. There are constraints on staff time and administrative considerations on Symphony, so there will be a support overhead for using the scanners. Uploading and running the programs does have to be managed, so staff will save the files and send them as an attachment to a named person, probably in the Stock Team. They can then schedule and run the programs, making sure they do not interfere with other programs that are already scheduled. Holding files on the PC have a limit of one file at a time and on Symphony you can have only one file per library. 1 file on scanner can have multiple sub-files.

These are the sorts of tasks we have in mind for the scanners:

- Frequent periodic checks for items marked MISSING or reserved items

- Annual Inventory of library, maybe rolling basis

- Search for items lost in transit

- Search for items reported lost

- Search for items added by EDI Order Fulfillment not in available stock

You do not actually have to upload the results every time, but it does help keep the catalogue clean and pick up anomalies. A Good Thing - BUT it takes time. Keeping the catalogue clean means customers and staff do not waste time looking for items the catalogue says we have but which in fact cannot be found. As some services are charged by catalogue size, having dead wood in there may be costing you money better spent elsewhere.

I did get a lot of comments about the hand-held scanners from staff »When are we getting one? « and from customers - one asked me »Are you disinfecting the books? «Staff were keen to get their hands on them and I got the feeling they'd rather search for missing or lost items with a scanner than with a huge sheaf of paper print outs.

How long does it take to search 5 or 6 thousand books subdivided into sections by genre for 248 items by eye with a sheaf of paper? It's a long time since I've done that, but I suspect a lot longer than 1 hour 40 minutes.

- Catherine Cooke_ccooke@westminster.gov.uk

- Intellident Ltd. http://www.bibliotheca.com

- SirsiDynix http://www.sirsidynix.com/products/symphony

- SmartSM http://www.smartsm.com/

This is my e-mail address in case anyone wants to follow any of this up and contact details for the UK arms of the companies we work with - I don't know their penetration in mainland Europe. Intellident as we have heard, merged with Bibliotheca earlier this year. 